\title{
On Properties of Uniformly Strongly Fuzzy Ideals
}

\author{
Flaulles B. Bergamaschi ${ }^{1}$, Alexsandra Oliveira Andrade ${ }^{1}$ and Regivan H. N. Santiago ${ }^{2}$ \\ 1. Department of Science and Technology, Southwest Bahia State University,Vitoria da Conquista, Bahia 45.031-900, Brazil \\ 2. Department of Informatics and Applied Mathematics, Federal University of Rio Grande do Norte, Natal 59.078-900, Rio Grande \\ do Norte, Brazil
}

\begin{abstract}
The main purpose of this paper is to continue the study of uniform strong primeness on fuzzy setting. A pure fuzzy notion of this structure allows us to develop specific fuzzy results on USP (uniformly strongly prime) ideals over commutative and noncommutative rings. Besides, the differences between crisp and fuzzy setting are investigated. For instance, in crisp setting an ideal $I$ of a ring $R$ is a USP ideal if the quotient $R / I$ is a USP ring. Nevertheless, when working over fuzzy setting this is no longer valid. This paper shows new results on USP fuzzy ideals and proves that the concept of uniform strong primeness is compatible with a-cuts. Also, the Zadeh's extension under epimorphisms does not preserve USP ideals. Finally, the t- and m- systems are introduced in a fuzzy setting and their relations with fuzzy prime and uniformly strongly prime ideals are investigated.
\end{abstract}

Key words: Fuzzy ideal, fuzzy set, uniformly strongly prime, prime.

\section{Introduction}

This paper begins with the following question: Is it possible to have a fuzzy version of a USP (uniformly strongly prime) ideal?

In 2014, Bergamaschi and Santiago answered this question by introducing in Ref. [1] the notion of USP fuzzy ideals without a-cut dependence for the first time. Since then we have been dedicated to investigate its properties and similarities with crisp setting. So this paper increases our understanding on USP ideals and fills the gap related with a-cuts as well as it introduces the concept of systems for a fuzzy environment.

The following paragraphs introduce the whole subject.

The importance of prime numbers for pure mathematics results and many applications is well-known.

For example, nowadays prime numbers are the fundamental idea behind cryptography. We may think of primeness for sets and subsets. In other words, it is

Corresponding author: Flaulles B. Bergamaschi, Ph.D., research field: fuzzy prime ideals.

**This research was partially supported by the Brazilian Research Council (CNPq) under the process 306876/2012-4. perfectly possible to define prime rings and prime ideals ${ }^{1}$.

A ring is a set endowed with two operations, addition and multiplication, where these operations have properties to those operations defined for the integers. Prime ideals are subsets of a ring with similar properties of prime numbers and they develop an important role in the study of rings as well as prime numbers in the ring of integers.

Prime rings are a special class of rings where the zero ideal (0) is a prime ideal. In a commutative ring theory prime rings are well-known as an integral domain i.e. rings where $a b=0$ implies $a=0$ or $b=0$.

The concept of SP (strongly prime) ring appeared in 1973 made by Lawrence in his Master's thesis as a subclass of prime rings. In 1975, Lawrence and Handelman [2] presented an extensive work on SP rings. They developed properties and proved important results, e.g., all prime rings may be embedded in an SP ring; all SP rings are nonsingular. Besides, they defined the USP ring as a subclass of SP rings and demonstrated some initial uniform

\footnotetext{
${ }^{1}$ If the reader has interest in applications about prime ideals and fuzzy prime ideals in coding theory it is worth reading the introduction of Ref. [14] and its references.
} 
properties.

After a little time, the study of USP rings became more important. Thus, in 1987, Olson [3] presented a paper about USP rings and USP radical. Olson proved that USP rings generate a radical class which properly contains both the right and left SP radicals and which is independent of the famous Jacobson and BrownMcCoy radicals. Also, some results in group ring theory were rediscovered by using the SP and USP ideals.

In 1965, Zadeh [4] introduced fuzzy sets and in 1971, Rosenfeld [5] introduced fuzzy sets in the realm of group theory and formulated the concept of fuzzy subgroups of a group. Since then, many researchers have been engaged in extending the concepts/results of abstract algebra to the broader framework of the fuzzy setting. Thus, in 1982, Liu [6] defined and studied fuzzy subrings as well as fuzzy ideals. Subsequently, Liu himself [7], Mukherjee and Sen [8], Swamy and Swamy [9], and Zhang Yue [10], among others, fuzzified certain standard concepts/results on rings and ideals. For example: Mukherjee was the first to study the notion of prime ideal in a fuzzy setting. Those studies were further carried out by Kumar in Refs. [11, 12], where the notion of nil radical and semiprimeness were introduced.

In 2013, motivated by crisp problems in group ring theory (e.g. isomorphism problem) we proposed in Ref. [13] the concept of SP ideal for fuzzy environment for the first time. The main goal was to investigate this structure in fuzzy environment. Thus, the concept of SP fuzzy ideal was born and it was called SPf ideal. The difficulty to find a pure fuzzy definition of an SP ideal forced us to define SPf ideals on cuts. But this approach had some issues. One of them lies in the fact that all results have similarities in crisp algebra. In other words, fuzzy setting became a mirror of classical theory.

Although a pure definition of SPf ideals was not founded, the ideas developed in Ref. [13] enabled us to create the definition of USPf (uniformly strongly prime fuzzy) ideal. The concept of USPf was possible because the crisp definition of USP ideal is more suitable to be translating to fuzzy environment. This approach became more interesting, since it allowed us to find pure fuzzy results. For instance, it was proved that every USPf ideal is a prime ideal according to the newest

Pure fuzzy definition of fuzzy prime ideal was given by Navarro [14] in 2012. Also, it was proved that some results in fuzzy setting are not true like in classical theory. Besides, the behavior of Zadeh's extension on USPf ideals was studied and as a consequence we built a version of correspondence theorem for USPf ideals.

This paper expands the last three papers $[1,13]$ with new properties of USPf ideals and introduces the $\mathrm{t}$ and m-systems for a fuzzy setting. It is shown that every fuzzy ideal is contained in a USPf ideal. Also, it is proved that the complement of prime/USP fuzzy ideal is an $\mathrm{m} / \mathrm{t}$-system. Moreover the concept of m-systems is compatible with the newest (2012) definition of fuzzy prime ideals over noncommutative rings given by Navarro et al. in Ref [14]. It is also shown that the inverse image of a USPf ideal is a USPf ideal when the mapping is a ring homomorphism extended by Zadeh's principle. However, on the direct image this is no longer valid. Finally, the paper leaves for the reader some open questions about USP investigation on fuzzy setting.

This paper has the following structure: Section 2, which not only provides an overview about the ring and fuzzy ring theory, but it also contains the classical definition and results of USP rings/ideals; Section 3 has the definition of USPf ideals and the compatibility with cuts; Section 4 shows that Zadeh's extension under epimorphisms does not preserve uniform strong primeness; Section 5 deals with $\mathrm{t}-\mathrm{m}$-systems and its relations with primeness and uniform strong primeness; Section 6 provides the final remarks.

\section{Preliminaries}

This section explains some definitions and results 
that will be required in the next sections. All rings are associative with identity and are usually denoted by $R$. Here, we expose primitive definitions of prime rings/ideals and Uniformly Strongly Prime rings/ideals.

Definition 1 A ring is a nonempty set $\mathrm{R}$ of elements closed under two binary operations + and $\cdot$ with the following properties:

$(\mathrm{R},+)$ (that is, the set $\mathrm{R}$ considered with the single operation of addition) is an abelian group (whose identity element is denoted $0_{R}$, or just 0 );

The operation $\bullet$ is associative: $\left(a^{\bullet} b\right)^{\bullet} \mathrm{c}=\mathrm{a}^{\bullet}(\mathrm{b} \bullet \mathrm{c})$ for every a, b, c e R. Thus, $(\mathrm{R}, \bullet)$ is a semigroup;

The operations + and - satisfy the two distribu- tive laws: $(a+b) \cdot c=a \cdot c+b \bullet c$ and $a \cdot(b+c)=a \cdot b+a \bullet c$, for every a, b, c $\in$ R.

If $R$ is a ring and there exists an element 1 such that $a \cdot 1=a$ for every $a$ e $R$ we say that the ring has multiplicative identity. Also, if $\mathrm{a} \bullet \mathrm{b}=\mathrm{b} \cdot \mathrm{a}$ for $\mathrm{a}, \mathrm{b} \in$ $\mathrm{R}$ we call $R$ a commutative ring.

Very often we omit writing the - for multiplication, that is, we write $a b$ to mean $a \cdot b$. Note that there can only be one additive identity in $\mathrm{R}$ (because $(\mathrm{R},+)$ is a group, and a group can only have one additive identity). Also, there can be only one multiplicative identity in $\mathrm{R}$. If $\mathrm{R}$ is commutative and for any $\mathrm{a}, \mathrm{b} \in$ $\mathrm{R}, \mathrm{ab}=0$ implies $\mathrm{a}=0$ or $\mathrm{b}=0$ we call $\mathrm{R}$ an integral domain. Note that the ring of $n \times n$ matrices with integers entries is a noncommutative ring and nor an integral domain.

Definition 2 Let R be a ring. A nonempty subset I of $\mathrm{R}$ is called a right ideal of $\mathrm{R}$ if:

(a) $\mathrm{a}, \mathrm{b} \in \mathrm{I}$ implies $\mathrm{a}+\mathrm{b} \in \mathrm{I}$;

(b) given $r \in \mathrm{Ra} \in \mathrm{I}$, then $\mathrm{ar} \in \mathrm{I}$ (that is, a right ideal absorbs right multiplication by the elements of the ring).

Similarly we can define left ideal replacing (b) by: (b') given $r \in R, a \in I$, then $r a \in I$. If $I$ is both right and left ideal of R, we call I a two-sided ideal or simply an ideal.

For the next definition consider: $\mathrm{IJ}=\left\{i_{1} j_{1}+\ldots+\right.$ $\mathrm{i}_{\mathrm{n}} \mathrm{j}_{\mathrm{n}}: \mathrm{i}_{\mathrm{k}} \in \mathrm{I}$ and $\mathrm{j}_{\mathrm{k}} \in \mathrm{J}, \mathrm{k}=1, \ldots, n$; where $\mathrm{n} \in$ $\left.\mathrm{Z}^{+}\right\}$and the set $\mathrm{x}$ Ry $=\{$ xry: $\mathrm{r} \in R\}$.

Definition 3 A prime ideal in an arbitrary ring $\mathrm{R}$ is any proper $(\mathrm{P} \subseteq \mathrm{R}$ and $\mathrm{P} \neq \mathrm{R}$ ) ideal $\mathrm{P}$ such that, whenever I, J are ideals of $\mathrm{R}$ with $\mathrm{IJ} \subseteq \mathrm{P}$, either $\mathrm{I} \subseteq \mathrm{P}$ or $\mathrm{J} \subseteq \mathrm{P}$.

Proposition 1 ([15], Proposition 10.2) An ideal $P$ of a ring $\mathrm{R}$ is prime if for $\mathrm{x}, \mathrm{y} \in \mathrm{R}, \mathrm{xRy} \subseteq \mathrm{P}$ implies $\mathrm{x}$ $\in \mathrm{P}$ or $\mathrm{y} \in \mathrm{P}$.

Definition 4 An ideal $\mathrm{P}$ of a ring $\mathrm{R}$ is called completely prime if given $a$ and $b$ two elements of $\mathrm{R}$ such that their product $a b \in \mathrm{P}$, then $a \in \mathrm{P}$ or $b \in \mathrm{P}$.

Given a ring $R$ and $a \in R$, the set $(a)=R a R=$ $\left\{\mathrm{x}_{1} a \mathrm{y}_{1}+\ldots+x_{n} a y_{n}: \quad \mathrm{n} \in \mathrm{N}, x_{i} y_{i} \in R\right\}$ is an ideal and is called the ideal generated by $a$.

Definition 5 Let A be a subset of a ring $\mathrm{R}$. The right annihilator of $A$ is defined as $\mathrm{An}_{\mathrm{r}}(\mathrm{A})=\{\mathrm{x} \in \mathrm{R}$ : $\mathrm{Ax}$ $=(0)\}$. Similarly, we can define the left annihilator of A.

Definition 6 [2] A ring $\mathrm{R}$ is called right strongly prime if for each nonzero $\mathrm{x} \in \mathrm{R}$ there exists a finite nonempty subset $F_{x}$ of $R$ such that the $A_{n}\left(x F_{x}\right)=$ (0).

When $R$ is right strongly prime we can prove that $\mathrm{F}_{\mathrm{x}}$ is unique and called a right insulator for $\mathrm{x}$. Handelman and Lawrence worked exclusively with rings with multiplicative identity. However, Parmenter, Stewart and Wiegandt [16] have shown that it is equivalent to:

Definition $7 \mathrm{~A}$ ring $\mathrm{R}$ is right strongly prime if each nonzero ideal $\mathrm{I}$ of $\mathrm{R}$ contains a finite subset $\mathrm{F}$ which has right annihilator zero.

It is clear that every right strongly prime ring is a prime ring. It is also possible to define left strongly prime in a manner analogous to that for right strong primeness.Handelman and Lawrence showed that these two concepts are distinct, by building a ring that is right strongly prime but not left strongly prime ([2], Example 1).

Example 1 Consider $Z_{n}$ the commutative ring of integers $\bmod n$, for $n>1$. If $a \in Z$, the class of $a$ is 
$[a]=\{x \in Z:(x \bmod n)=a\}$. Note that if $n$ is not a prime number, then there exists $p, q \in Z$ such that $n$ $=\mathrm{p} \mathrm{q}$, where $0<\mathrm{p}<\mathrm{n}$ and $0<\mathrm{q}<\mathrm{n}$. Hence, $[\mathrm{p} \mathrm{q}]=0$ in $Z_{n}$, but $[p] \neq 0$ and $[q] \neq 0$. We conclude that $Z_{n}$ is not a integral domain and as a consequence $Z_{n}$ is not a prime ring. Thus, $Z_{n}$ is not right strongly prime ring. On the other hand, if $n$ is prime, $Z_{n}$ is a field, hence right strongly prime ring.

Definition 8 A ring is a bounded right strongly prime ring of bound $n$, if each nonzero element has an insulator containing no more than $n$ elements and at least one element has no insulator with fewer than $n$ elements.

Definition 9 A ring is called uniformly right strongly prime if the same right insulator may be chosen for each nonzero element.

Since an insulator must be finite, it is clear that every uniformly strongly prime ring is a bounded right strongly prime ring of bound $n$. Again, analogous definitions of bounded left strongly prime and uniformly left strongly prime can be formulated. As was the case with the notation of strong primeness it is possible to find rings which are bounded left strongly prime but not bounded right strongly prime, and vice-versa (see Ref. [2], Example 1). However, Olson [3] showed that the concept of uniformly strongly prime ring is two-sided in view of the following result:

Lemma 1 [3] A ring $R$ is right/left uniformly strongly prime if there exists a finite subset $F \subseteq R$ such that for any two nonzero elements $\mathrm{x}$ and $\mathrm{y}$ of $\mathrm{R}$, there exists $\mathrm{f} \in \mathrm{F}$ such that $\mathrm{xfy} \neq 0$.

Corollary 2 [3] R is uniformly right strongly prime ring if and only if $\mathrm{R}$ is uniformly left strongly prime ring.

Lemma 3 [3] The following are equivalent:

(i) R is a uniformly strongly prime ring;

(ii) There exists a finite subset $\mathrm{F} \subseteq \mathrm{R}$ such that $\mathrm{xFy}$ $=0$ implies $\mathrm{x}=0$ or $\mathrm{y}=0$, where $\mathrm{x}, \mathrm{y} \in \mathrm{R}$;

Definition 10 An ideal I of a ring $\mathrm{R}$ is called USP ideal if there exists a finite set $\mathrm{F} \subseteq \mathrm{R}$ such that for two nonzero elements $\mathrm{x}$ and $\mathrm{y}$ of $\mathrm{R} / \mathrm{I}$ (the complement of I in $\mathrm{R}$ ), there exists $\mathrm{f} \in \mathrm{F}$ such that $\mathrm{xfy} \notin \mathrm{I}$.

Proposition 2 An ideal I of a ring R is a USP ideal if the quotiente $\mathrm{R} / \mathrm{I}$ is a USP ring.

Proposition 3 An ideal I of a ring R is a USP if there exists a finite set $\mathrm{F} \subseteq \mathrm{R}$ such that $\mathrm{xFy} \subseteq \mathrm{I}$ implies $\mathrm{x} \in \mathrm{I}$ or $\mathrm{y} \in \mathrm{I}$, where $\mathrm{x}, \mathrm{y} \in \mathrm{R}$.

From this point forward consider $\wedge$ as infimum and $\vee$ as supremum in $[0,1]$.

Definition 11 A fuzzy subset I: $\mathrm{R} \rightarrow[0,1]$ of a ring $\mathrm{R}$ is called a fuzzy subring of $\mathrm{R}$ if for all $\mathrm{x}, \mathrm{y} \in \mathrm{R}$ the following requirements are met:

(1) $\mathrm{I}(\mathrm{x}-\mathrm{y}) \geq \mathrm{I}(\mathrm{x}) \wedge \mathrm{I}(\mathrm{y})$;

(2) $\mathrm{I}(\mathrm{xy}) \geq \mathrm{I}(\mathrm{x}) \wedge \mathrm{I}(\mathrm{y})$;

If condition (2) is replaced by

$\mathrm{I}(\mathrm{xy}) \geq \mathrm{I}(\mathrm{x}) \vee \mathrm{I}(\mathrm{y})$, then I is called a fuzzy ideal of $\mathrm{R}$.

Note that using properties of the t-norm we have for any fuzzy subring/fuzzy ideal $I$ of a ring $R$ the following: if for some $\mathrm{x}, \mathrm{y} \in \mathrm{R}, \mathrm{I}(\mathrm{x})<\mathrm{I}(\mathrm{y})$, then $\mathrm{I}(\mathrm{x}$ $-\mathrm{y})=\mathrm{I}(\mathrm{x})=\mathrm{I}(\mathrm{y}-\mathrm{x})$; Also, if $I$ is a fuzzy ideal of a ring $\mathrm{R}$, then $\mathrm{I}(1) \leq \mathrm{I}(\mathrm{x}) \leq \mathrm{I}(0)$ for all $\mathrm{x} \in R$.

Given a fuzzy set $I$ and $a$ in $[0,1]$ the set $I_{a}=\{x \in R$; such that $\mathrm{I}(\mathrm{x}) \geq \mathrm{a}$ \} is called a-cut of $\mathrm{I}$.

Proposition 4 [4] A fuzzy subset I of a ring $\mathrm{R}$ is a fuzzy ideal of $R$ if all a-cuts $I_{a}$ are ideals of R.

Definition 12 (Zadeh's Extension) Let $\mathrm{f}$ be a function from set $\mathrm{X}$ into $\mathrm{Y}$, and let $\mu$ be a fuzzy subset of $\mathrm{X}$. Define the fuzzy subset $\mathrm{f}(\mu)$ of $\mathrm{Y}$ in the following way: For all $\mathrm{y} \in \mathrm{Y}$,

$$
f(\mu)(y)=\left\{\begin{array}{c}
\vee\{\mu(x): x \in X, f(x)=y\}, \\
i f f^{-1}(y) \neq \emptyset \\
0, \quad \text { otherwise. }
\end{array}\right.
$$

If $\lambda$ is a fuzzy subset of $\mathrm{Y}$, we define the fuzzy

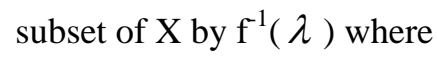

$\mathrm{f}^{-1}(\lambda)(\mathrm{x})=\lambda(\mathrm{f}(\mathrm{x}))$.

Definition 13 [14] Let $\mathrm{R}$ be a ring with unity. A nonconstant fuzzy ideal $P: R \rightarrow[0,1]$ is said to be prime or fuzzy prime ideal if for any $x, y \in R, \wedge$ $\mathrm{P}(\mathrm{xRy})=\mathrm{P}(\mathrm{x}) \vee \mathrm{P}(\mathrm{y})$.

Proposition5 [14] Let R be an arbitrary ring with 
unity and $P: R \rightarrow[0,1]$ be a nonconstant fuzzy ideal of $\mathrm{R}$. The following conditions are equivalent:

(i) $\mathrm{P}$ is prime;

(ii) $\mathrm{P}_{\mathrm{a}}$ is prime for all $\mathrm{P}(1)<\mathrm{a} \leq \mathrm{P}(0)$;

(iii) $\mathrm{R} / \mathrm{P}_{\mathrm{a}}$ is a prime ring for all $\mathrm{P}(1)<\mathrm{a} \leq \mathrm{P}(0)$;

(iv) For any fuzzy ideal J, if $\mathrm{J}$ (xry) $\leq \mathrm{P}$ (xry) for all $\mathrm{r} \in \mathrm{R}$, then $\mathrm{J}(\mathrm{x}) \leq \mathrm{P}(\mathrm{x})$ or $\mathrm{J}(\mathrm{y}) \leq$ $\mathrm{P}(\mathrm{y})$.

Let $I$ be a fuzzy ideal of a ring R. For all $r \in R$ define fuzzy left coset $\mathrm{r}+1$, where $(\mathrm{r}+1)(\mathrm{x})=\mathrm{I}(\mathrm{x}-\mathrm{r})$.

Given an ideal (crisp or fuzzy) of $R$ define $\mathrm{R} / \mathrm{I}=\{\mathrm{r}$ +1 : r e R $\}$ the quotient ring by $I$. In $R / I$ we can define + and $\bullet$, where $(\mathrm{r}+\mathrm{I})+(\mathrm{s}+\mathrm{I})=(\mathrm{r}+\mathrm{s})+\mathrm{I}$ and $(\mathrm{r}+\mathrm{I}) \cdot$ $(\mathrm{s}+\mathrm{I})=(\mathrm{rs})+\mathrm{I}$.

Proposition 6 [3] If $\mathrm{I}$ and $\mathrm{P}$ are ideals of a ring $\mathrm{R}$ with $\mathrm{P}$ a USP ideal, then $\mathrm{I} \cap \mathrm{P}$ is a USP ideal.

\section{Uniform Strong Primeness}

This section introduces the concept of Uniformly Strongly Prime fuzzy ideal (or shortly USPf ideal) according to definition given in Ref. [1].

Definition 14 [1] Let R be an associative ring with unity. A non-constant fuzzy ideal $\mathrm{I}: \mathrm{R} \rightarrow[0,1]$ is said to be Uniformly Strongly Prime fuzzy (USPf) ideal if there exists a finite subset $\mathrm{F}$ such that $\wedge \mathrm{I}(\mathrm{xFy})=$ $\mathrm{I}(\mathrm{x}) \vee \mathrm{I}(\mathrm{y})$, for any $\mathrm{x}, \mathrm{y} \in \mathrm{R}$. The set $\mathrm{F}$ is called insulator of I.

Proposition 7 Let $\mathrm{R}$ be an associative ring with unity and Aa crisp USP ideal of R. Then I, where $\mathrm{I}(\mathrm{x})=1$ if $\mathrm{x}$ in $\mathrm{A}$, and $\mathrm{I}(\mathrm{x})=0$ otherwise, is a USPf ideal of R.

Proof Clearly $I$ is a fuzzy ideal. According to definition 10 given $\mathrm{x}, \mathrm{y} \in \mathrm{R}$ there exists a finite set $F \subseteq R$ such that if $\mathrm{xFy} \subseteq A$ implies $\mathrm{x} \in \mathrm{A}$ or $\mathrm{y} \in \mathrm{A}$. Suppose $\mathrm{xFy} \subseteq \mathrm{A}$, then $\wedge \mathrm{I}(\mathrm{xFy})=1=\mathrm{I}(\mathrm{x}) \vee \mathrm{I}(\mathrm{y})$. If $\mathrm{xFy} \nsubseteq \mathrm{A}$, then there exists $\mathrm{f} \in \mathrm{F}$ such that $\mathrm{xfy} \notin$ A. Hence, $\mathrm{x}, \mathrm{f}, \mathrm{y} \notin \mathrm{A}$. Therefore, $\wedge \mathrm{I}(\mathrm{xFy})=0=\mathrm{I}(\mathrm{x})$ $\checkmark \mathrm{I}(\mathrm{y})$.

Proposition $8 \mathrm{I}$ is USPf ideal of R if $\mathrm{I}_{\mathrm{a}}$ is USP ideal of $\mathrm{R}$ for all $\mathrm{I}(1)<\mathrm{a}<\mathrm{I}(0)$.

Proof Suppose Ia USPf ideal and let $F \subseteq R$ be a finite set given by definition 14. Let $\mathrm{x}, \mathrm{y} \in \mathrm{R}$ and $\mathrm{I}(1)<\mathrm{a}<\mathrm{I}(0)$ such that $\mathrm{xFy} \subseteq$ Ia. Hence, $\mathrm{I}(\mathrm{x}) \vee$ $\mathrm{I}(\mathrm{y})=\wedge \mathrm{I}(\mathrm{xFy}) \geq \mathrm{a}$, and thus $\mathrm{I}(\mathrm{x}) \geq \mathrm{a}$ or $\mathrm{I}(\mathrm{y}) \geq \mathrm{a}$. Therefore, $\mathrm{x} \in \mathrm{I}_{\mathrm{a}}$ or $\mathrm{y} \in \mathrm{I}_{\mathrm{a}}$. On the other hand, suppose $\mathrm{I}_{\mathrm{a}}$ is a USP ideal of $\mathrm{R}$ for all $\mathrm{I}(1)<\mathrm{a} \leq \mathrm{I}(0)$. According to proposition 3 each $I_{a}$ has a finiteset $F_{a}$ such that if $\mathrm{xF}_{\mathrm{a}} \mathrm{y} \subseteq \mathrm{I}_{\mathrm{a}}$ implies $\mathrm{x} \in \mathrm{I}_{\mathrm{a}}$ or $\mathrm{y} \in \mathrm{I}_{\mathrm{a}}$. Let a finite set $\mathrm{F}=\bigcap \mathrm{F}_{\mathrm{a}}$. Suppose $\mathrm{I}(1)<\mathrm{a}<\mathrm{I}(0)$ and $\wedge$ $\mathrm{I}(\mathrm{xFy})>\mathrm{I}(\mathrm{x}) \vee \mathrm{I}(\mathrm{y})$ and $\mathrm{t}=\wedge \mathrm{I}(\mathrm{xFy})$ for any $\mathrm{x}, \mathrm{y} \in$ R. Note that $\mathrm{t}>\mathrm{I}(\mathrm{x}) \vee \mathrm{I}(\mathrm{y})$ and $\mathrm{t} \leq \mathrm{I}$ (xfy) for all $\mathrm{f} \in \mathrm{F}$. Hence, $\mathrm{x}, \mathrm{y} \notin \mathrm{I}_{\mathrm{t}}$, but $\mathrm{xFy} \subseteq \mathrm{I}_{\mathrm{t}}$ and thus (by hypothesis) $\mathrm{x} \in \mathrm{I}_{\mathrm{t}}$ or $\mathrm{y} \in \mathrm{I}_{\mathrm{t}}$, where we have a contradiction. Therefore, $\wedge \mathrm{I}(\mathrm{xFy})=\mathrm{I}(\mathrm{x}) \vee \mathrm{I}(\mathrm{y})$.

Corollary 4 If $I$ is a USPf ideal of a ring R if R/I is a USP ring for all $\mathrm{I}(1)<\mathrm{a} \leq \mathrm{I}(0)$.

Corollary5 If I is USPf ideal, then I is fuzzy prime ideal.

For the following result $\operatorname{Ker}(\mathrm{f})=\{\mathrm{x} \in \mathrm{R}: \mathrm{f}(\mathrm{x})=0\}$ is the kernel of the homomorphism $f$ and $f^{-1}(J)$ is the fuzzy subset of R by Zadeh's extension.

Proposition9 [1] If $f: R \rightarrow S$ is a homomorphism of rings and J USPf ideal of S, then $\mathrm{f}^{-1}(\mathrm{~J})$ is USPf ideal of $R$ which is constant on $\operatorname{Ker}(\mathrm{f})$.

After proposition 9 we can think about the direct image. In other words, if I is a USPf ideal of R which is constant on $\operatorname{Ker}(f)$, then $f(I)$ is USPf is an ideal? In this paper we proved this statement as false, according to proposition 13 in the next section.

For the next result consider $\mathrm{I}_{*}=\mathrm{I}_{\mathrm{I}(0)}=\{\mathrm{x} \in \mathrm{R}$ : $\mathrm{I}(\mathrm{x})$ $=\mathrm{I}(0)\}$.

Proposition 10 [1] If I is a USPf ideal of a ring R, then $\mathrm{R} / \mathrm{I} * \mathrm{R} / \mathrm{I}$.

Corollary 6 [1] If $\mathrm{f}: \mathrm{R} \rightarrow \mathrm{S}$ is an epimorphism and I USPf ideal of $\mathrm{R}$ which is constant on $\operatorname{Ker}(\mathrm{f})$, then $\mathrm{R} / \mathrm{I} \cong \mathrm{S} / \mathrm{f}(\mathrm{I})$.

Proposition 11 [1] Let $\mathrm{J}$ be a crisp ideal of $\mathrm{R}$. Define I: $R \rightarrow[0,1]$ as $\mathrm{I}(\mathrm{x})=1$ if $\mathrm{x}=0, \mathrm{I}(\mathrm{x})=\mathrm{a}$ if $\mathrm{x} \in \mathrm{J} \backslash\{0\}, \mathrm{I}(\mathrm{x})=0$ if $\mathrm{x} \notin \mathrm{J}$, where $\mathrm{a} \in(0,1)$.

Then:

(i) I is a fuzzy ideal;

(ii) I is a USPf ideal if $\mathrm{J}$ is USP ideal. 
Corollary 7 Let $I$ be a nonconstant fuzzy ideal of $R$, and define $M(x)=I(0)$ if $x=0, M(x)=\alpha$ if $x \in I * \backslash\{0\}$, $\mathrm{M}(\mathrm{x})=\mathrm{I}(1)$ if $\mathrm{x} \notin \mathrm{I} *$. Then, $\mathrm{M}$ is a USPf ideal of $\mathrm{R}$ if $\mathrm{I} *$ is a USP ideal of $R$.

Corollary 8 Let $I$ a fuzzy ideal of a ring $R$ and $\operatorname{Im}(\mathrm{I})$ is a finite set. Then, $I$ is a USPf ideal if $I_{a}$ is a USP ideal for all $\mathrm{I}(1)<\mathrm{a} \leq \mathrm{I}(0)$.

Example 2 Consider $\mathrm{Z}$ the ring of integers and $4 \mathrm{Z}=$ $\{x \in Z: x=4 q, q \in Z\}$. Define $I(x)=1$ if $x=0$, $I(x)=1 / 2$ if $x \in 4 Z \backslash\{0\}, I(x)=0$ if $x \notin 4 Z$. It is easy to see that $\mathrm{I}$ is a fuzzy ideal, since its all a-cuts $\left(\mathrm{I}_{1}=(0)\right.$, $\mathrm{I}_{1 / 2}=4 \mathrm{Z}, \mathrm{I}_{0}=\mathrm{Z}$ ) are ideals. Moreover, $\mathrm{I}$ is not USPf ideal, since $4 \mathrm{Z}$ is not prime ideal, acccording to proposition 11 . Note that $\mathrm{I}_{*}=(0)$ is USP ideal. Hence, $\mathrm{R} / \mathrm{I} *$ is a USP ring. Applying the proposition $10 \mathrm{R} / \mathrm{I} \cong$ $\mathrm{R} / \mathrm{I}$ * Therefore, $\mathrm{R} / \mathrm{I}$ is a USP ring, although $\mathrm{I}$ is not a USPf ideal.

The next example shows us that direct image of USPf ideals by Zadeh's extension on homomorphism are not USPf ideals.

Example 3 [1] Let $\mathrm{f:} \mathrm{Z} \rightarrow \mathrm{Z}_{4}$ be defined by $\mathrm{f}(\mathrm{x})=$ $|x|_{4}=\mathrm{x}$ mod 4. Then, the function $\mathrm{f}$ is an epimorphism with kernel 4Z. Consider $\mathrm{I}(\mathrm{x})=1$ if $\mathrm{x}=0$, $I(x)=1 / 2$ if $x \in 3 Z \backslash\{0\}, I(x)=0$ if $x \notin 3 Z$. Also consider $f(\mathrm{I})(\mathrm{y})=1$ if $\mathrm{x}=0, \mathrm{f}(\mathrm{I})(\mathrm{y})=1 / 2$ if $\mathrm{x} \neq 0$. Clearly $\mathrm{I}$ is USPf ideal of $\mathrm{Z}$, but $\mathrm{f}(\mathrm{I})$ is not a USPf ideal of $\mathrm{Z}_{4}$, since $\mathrm{I}_{1 / 2}=\mathrm{Z}_{4}$ is not a USP ideal.

\section{Uniform Strong Primeness under Homomorphism}

This section amplifies results about USPf ideals. The first one (proposition 12) is geared to commutative rings. But it may be valid in noncommutative case (conjecture 2). The proposition 3 shows the difference between crisp and fuzzy setting by showing the behavior of Zadeh's extension on USPf ideals.

Proposition 12 If I is a nonconstant fuzzy ideal of a commutative ring $\mathrm{R}$, then there exists a USPf ideal $\mathrm{K}$ such that $\mathrm{I} \subseteq \mathrm{K}$.
Consider the ideal $\mathrm{I}^{*}=\{\mathrm{x} \in \mathrm{R}: \mathrm{I}(\mathrm{x})>\mathrm{I}(1)\}$, by Zorn's Lemma, there exists a maximal ideal $M$ of $\mathrm{R}$ containing $\mathrm{I}$. Now we can define the following fuzzy set: $\mathrm{K}(\mathrm{x})=\mathrm{I}(0)$ if $\mathrm{x} \in \mathrm{M}, \mathrm{K}(\mathrm{x})=\mathrm{I}(1)$ if $\mathrm{x} \notin \mathrm{M}$. Clearly, $\mathrm{K}$ is a fuzzy ideal and $\mathrm{I} \subseteq \mathrm{K}$. Now, consider the finite set $F=\{1\}$. Thus, $\wedge \mathrm{K}(\mathrm{xFy})=\mathrm{K}(\mathrm{xy})$ for any $\mathrm{x}, \mathrm{y}$ $\in \mathrm{R}$. If $\mathrm{x} \in \mathrm{M}$ or $\mathrm{y} \in \mathrm{M}$, then $\mathrm{xy} \in \mathrm{M}$ and then $\mathrm{K}(\mathrm{xy})=\mathrm{I}(0)=\mathrm{K}(\mathrm{x}) \vee \mathrm{K}(\mathrm{y})$. On the other hand, as $\mathrm{R}$ is commutative, $\mathrm{M}$ is completely prime, hence if $\mathrm{x} \notin$ $\mathrm{M}$ and $\mathrm{y} \notin \mathrm{M}$, then $\mathrm{xy} \notin \mathrm{M}$. Therefore, $\mathrm{K}(\mathrm{xy})=\mathrm{I}(1)$ $=\mathrm{I}(1) \vee \mathrm{I}(1)=\mathrm{K}(\mathrm{x}) \vee \mathrm{K}(\mathrm{y})$.

Conjecture 1 According to definition of fuzzy maximal ideal given by Malikin [17], Kin the demonstration of proposition 12 is a fuzzy maximal ideal.

Conjecture 2 The propostion 12 can be extended to noncommutative rings.

Proposition 13 Let $\mathrm{f}: \mathrm{R} \rightarrow \mathrm{S}$ is a epimorphism of commutative and not USP rings. If I is a USPf ideal of $R$ which is constant on $\operatorname{Ker}(f)$, then $f(I)$ is not a USPf ideal of S.

Proof As I is constant on $\operatorname{Ker}(f)$, then by Proposition 10 and Corollary 6 we have: $\mathrm{R} / \mathrm{I}_{*} \cong \mathrm{R} / \mathrm{I}$ $\cong \mathrm{R} / \mathrm{f}(\mathrm{I}) \cong \mathrm{R} / \mathrm{f}(\mathrm{I}) *$. As $\mathrm{I} *$ is USP ideal, then $\mathrm{R} / \mathrm{I}$ * is USP ring. Hence, $\mathrm{R} / \mathrm{f}(\mathrm{I}) *$ is USP ring. Thus, $\mathrm{f}(\mathrm{I}) *$ is USP ideal. As we know $\mathrm{f}(\mathrm{I}) * \subseteq \mathrm{f}(\mathrm{I})_{\mathrm{a}}$ for all a $\in$ $[0,1]$. But $\mathrm{S}$ is commutative and $\mathrm{f}(\mathrm{I}) *$ is Prime, hence $\mathrm{f}$ $(\mathrm{I}) *$ is maximal, this last statement implies $\mathrm{f}(\mathrm{I})_{\mathrm{a}}=\mathrm{S}$ for all a $\neq \mathrm{I}(0)$ and by hypoteses $S$ is not USP ring. Therefore, $\mathrm{f}(\mathrm{I})$ is not a USPf ideal.

Question 1 The proposition 13 shows us that USPf ideals cannot preserved by Zadeh's extension. Thus, we ask: Under which conditions can Zadeh's extension preserves the USPf ideals?

Proposition 14 If $I$ and $\mathrm{P}$ are fuzzy ideals of a ring $\mathrm{R}$ with $\mathrm{P}$ is a USPf ideal, then $\mathrm{I} \cap \mathrm{P}$ is a USPf ideal of $R$.

Proof Note that: $\wedge(\mathrm{I} \cap \mathrm{P})(\mathrm{xFy})=(\wedge \mathrm{I}(\mathrm{xFy})) \wedge$ $(\wedge \mathrm{P}(\mathrm{xFy}))=(\wedge \mathrm{I}(\mathrm{xFy})) \wedge(\mathrm{P}(\mathrm{x})) \vee \mathrm{P}(\mathrm{y}) \leq \mathrm{P}(\mathrm{x})$ $\checkmark \mathrm{P}(\mathrm{y})$.

Proposition 15 Any USPf ideal contains properly 
another USPf ideal.

Proof Suppose $I$ USPf ideal of a ring R. Let $P=\frac{1}{2} I$ $\subset$ I defined by $P(x)=\frac{1}{2} I(x)$ for all $x \in$ R. Hence, $\wedge P(x F y)=\wedge \frac{I(x F y)}{2}=\frac{I(x)}{2} \vee \frac{I(y)}{2}=P(x) \vee P(y)$

The following proposition tell us about the following question: If a fuzzy ideal has at least one USP a-cut, what can we say about this ideal. Is it a USPf ideal?

Proposition 16 Let $I$ is a nonconstant fuzzy Ideal of a integral domain $\mathrm{R}$ and $\mathrm{R}$ is not a USP ring and $\mathrm{I}_{\mathrm{t}}$ is USP ideal for some I (1) $<\mathrm{t} \leq \mathrm{I}(0)$. If $\mathrm{k} \neq \mathrm{t}$ and $\mathrm{I}_{\mathrm{k}}$ $\neq I_{t}$, then $I_{k}$ is not a USP ideal. Hence, $I$ is not a USPf ideal.

Proof When $I_{k}=R$ is trivial. Now suppose $I_{k} \neq R$ and note that in a integral domain if I is a USP ideal, then $\mathrm{I}$ is a Maximal ideal. Thus, $\mathrm{I}_{\mathrm{t}} \subset \mathrm{I}_{\mathrm{k}}$ is impossible, since it is Maximal. If $\mathrm{I}_{\mathrm{k}} \subset \mathrm{I}_{\mathrm{t}}$ implies $\mathrm{I}_{\mathrm{k}}$ not maximal, then $I_{k}$ not a USP ideal.

\section{The Fuzzy m- and t-Systems}

An m-system is a generalization of multiplicative systems. In the ring theory a set $\mathrm{M}$ is an m-system if for any two elements $\mathrm{x}, \mathrm{y}$ in $\mathrm{M}$ there exists $\mathrm{r}$ in $\mathrm{R}$ such that the product xry belongs to $\mathrm{M}$. It is not hard to perceive that an ideal is prime if its complement is an m-system (Mccoy [18]). On the other hand we have the t-systems which are sets where given any two elements $\mathrm{x}, \mathrm{y}$ in $\mathrm{T}$ there exists a finite set $\mathrm{F}$ such that xfy belongs to $\mathrm{T}$ for some $\mathrm{f}$ in $\mathrm{F}$. Clearly a t-system is an m-system. Olson [3] proved that I is a uniformly strongly prime ideal if its complement is a t-system.

In this section we will introduce the m-systems in a fuzzy setting based on the definition of fuzzy prime ideals defined by Navarro [14] in 2012. The fuzzy tsystems are also introduced. As we shall soon see it is possible to prove that an ideal I is a fuzzy prime ideal if its complement is an m-system. Moreover, I is USPf ideal if its complement is a t-system.

Definition 15 [18] A subset $M$ of a ring $R$ is called an m-system if for any two elements $\mathrm{x}, \mathrm{y} \in \mathrm{M}$ there exists $r \in \mathrm{R}$ such that $\mathrm{xry} \in \mathrm{M}$.

Definition 16 [3] A subset $\mathrm{T}$ of a ring $\mathrm{R}$ is called a t-system if there exists a finite set $\mathrm{F} \subseteq R$ such that for any two elements $x, y \in T$ there exists $f \in F$ such that $x f y \in T$.

It is not hard to prove that $\mathrm{F}$ is unique. So, it will be called the insulator of $\mathrm{T}$. Note that, the empty set will be a t-system.

Proposition 17 [18] If $\mathrm{M}$ is a t-system, then $\mathrm{M}$ is a m-system.

Proposition 18 [18] I is a prime ideal of a ring $\mathrm{R}$ if $\mathrm{R} \backslash \mathrm{I}$ (the complement of $\mathrm{I}$ in $\mathrm{R}$ ) is an m-system.

Proposition 19 [3] An ideal I is a USP ideal of a ring $\mathrm{R}$ if $\mathrm{R} \backslash \mathrm{I}$ (the complement of $\mathrm{I}$ in $\mathrm{R}$ ) is a t-system.

For the following definition consider $\mathrm{xRy}=\{\mathrm{xry}: \mathrm{r}$ $\in \mathrm{R}\}$.

Definition 17 Let $\mathrm{R}$ be an associative ring with unity. A nonconstant fuzzy set $\mathrm{K}: \mathrm{R} \rightarrow[0,1]$ is said to be a fuzzy m-system if $\vee K(x R y)=K(x) \wedge$ $\mathrm{K}(\mathrm{y})$, for any $\mathrm{x}, \mathrm{y} \in \mathrm{R}$.

Proposition 20 If $\mathrm{K}$ is a fuzzy subset of a ring $\mathrm{R}$ such that $\mathrm{K}_{\mathrm{a}}$ is an m-system for all a-cuts, then

$\vee K(\mathrm{xRy}) \geq \mathrm{K}(\mathrm{x}) \wedge \mathrm{K}(\mathrm{y})$,

Proof Let $x, y \in R$ and $t=K(x) \wedge K(y)$. As $K_{t}$ is an m-system and $x, y \in \mathrm{K}_{\mathrm{t}}$ then there exists $\mathrm{r} \in \mathrm{R}$ such that $x r y \in$ Kti.e $K(x r y) \geq$ t. Hence, $\vee K(x$ R y) $\geq \mathrm{t}$.

Question 2 Under which conditions may we have the following result: $\mathrm{K}$ is a fuzzy m-system of $\mathrm{R}$ if $\mathrm{K}_{\mathrm{a}}$ is a m-system for all a-cuts?

For the following results consider $P$ the fuzzy ideal and $\mathrm{P}_{\mathrm{C}}=1-\mathrm{P}$ the complement of $\mathrm{P}$ in $\mathrm{R}$.

Corollary $9 \mathrm{P}$ is a fuzzy prime ideal of $\mathrm{R}$ if $\mathrm{P}_{\mathrm{C}}$ is a fuzzy m-system.

Proof Suppose $P$ fuzzy prime, then

$\wedge P(x R y)=\mathrm{P}(\mathrm{x}) \vee \mathrm{P}(\mathrm{y})$ for any $\mathrm{x}, \mathrm{y} \in \mathrm{R}$. Hence, $\checkmark P_{c}(x R y)=V(1-P(x R y))=1-\wedge P(x R y)=1-(P(x)$ $\vee \mathrm{P}(\mathrm{y}))=(1-\mathrm{P}(\mathrm{x})) \wedge(1-\mathrm{P}(\mathrm{y}))=\mathrm{I}_{\mathrm{c}}(\mathrm{x}) \wedge \mathrm{I}_{\mathrm{c}}(\mathrm{y})$. Suppose now $\mathrm{P}_{\mathrm{c}}$ is a fuzzy m-system, then $V \mathrm{P}_{\mathrm{c}}(\mathrm{x} R \mathrm{y})$ $=\mathrm{P}_{\mathrm{c}}(\mathrm{x}) \wedge \mathrm{P}_{\mathrm{c}}(\mathrm{y})$ for any $\mathrm{x}, \mathrm{y} \in \mathrm{R}$. Thus, $1-\wedge \mathrm{P}(\mathrm{x} R \mathrm{y})$ $=1-(\mathrm{P}(\mathrm{x}) \vee \mathrm{P}(\mathrm{y}))$. Therefore $\wedge \mathrm{P}(\mathrm{x} R \mathrm{y})=\mathrm{P}(\mathrm{x}) \vee \mathrm{P}(\mathrm{y})$.

For the following definition consider the subset 
$\mathrm{xFy}=\{\mathrm{xfy}: \mathrm{f} \in \mathrm{F}\}$ of ring $R$.

Definition 18 Let $\mathrm{R}$ be an associative ring with unity. A nonconstant fuzzy set $\mathrm{M}: \mathrm{R} \rightarrow[0,1]$ is said to be a fuzzy t-system if there exists a finite subset $F$ such that $\vee M(x F y)=M(x) \wedge M(y)$, for any $x, y \in R$.

Proposition $21 \mathrm{I}$ is a USPf ideal of $\mathrm{R}$ if $\mathrm{I}_{\mathrm{C}}$ (the complement of I in R) is a fuzzy t-system.

Proof Suppose $I$ USPf, then there exists a finite set F where $\wedge \mathrm{I}(\mathrm{xFy})=\mathrm{I}(\mathrm{x}) \vee \mathrm{I}(\mathrm{y})$ for any $\mathrm{x}, \mathrm{y} \in R$. Hence, $\vee I_{\mathrm{c}}(\mathrm{xFy})=\vee(1-I(\mathrm{xFy}))=1-\wedge I(\mathrm{x} F \mathrm{y})=1-$ $(\mathrm{I}(x) \vee \mathrm{I}(y))=(1-\mathrm{I}(x)) \wedge(1-\mathrm{I}(\mathrm{y}))=\mathrm{I}_{\mathrm{c}}(\mathrm{x}) \wedge \mathrm{I}_{\mathrm{c}}(\mathrm{y})$. Suppose now $I_{C}$ is a fuzzy $t$-system, then there exists a finite set $F$ where $\vee \mathrm{I}_{\mathrm{c}}(\mathrm{xFy})=\mathrm{I}_{\mathrm{c}}(\mathrm{x}) \wedge \mathrm{I}_{\mathrm{c}}(\mathrm{y})$ for any $\mathrm{x}, \mathrm{y}$ $\in \mathrm{R}$. Thus, $1-\wedge \mathrm{I}(\mathrm{xFy})=1-(\mathrm{I}(\mathrm{x}) \vee \mathrm{I}(\mathrm{y}))$. Therefore $\wedge \mathrm{I}(\mathrm{x} F \mathrm{Fy})=\mathrm{I}(\mathrm{x}) \vee \mathrm{I}(\mathrm{y})$.

Proposition 22 If $\mathrm{M}$ is a fuzzy t-system of $\mathrm{R}$, then $\mathrm{M}_{\mathrm{a}}$ is a t-system for all a-cuts.

Proof As M is a fuzzy t-system there exists a finite set $F$, where $\wedge \mathrm{I}(\mathrm{x} F \mathrm{y})=\mathrm{I}(\mathrm{x}) \vee \mathrm{I}(\mathrm{y})$ for any $\mathrm{x}, \mathrm{y} \in \mathrm{R}$. Let $x, y \in M_{a}$, then $\vee M(x F y)=M(x) \wedge M(y) \geq a$. Since $F$ is a finite set, there exists $f \in F$ such that $M$ (xfy) $>$ a. Thus, xfy $\in M_{a}$. Therefore, $M_{a}$ is a t-system.

Question 3 Under which conditions may we have the following result: If $\mathrm{T}$ is a fuzzy t-system of $\mathrm{R}$, then $\mathrm{T}$ is an m-system?

\section{Conclusion}

Prime ideals are structural pieces of a ring and should be the first part in the study of its properties. As it is known we can decompose an ideal in the product of prime ideals. Thus, the following question is immediate: may we decompose a fuzzy ideal in a product of fuzzy prime ideals? To answer this question we need to first of all understand primeness in fuzzy setting. So, this paper contributes to this end and also develops some thoughts on fuzzy ring theory.

\section{Acknowledgment}

The authors would like to thank UESB (Southwest Bahia State University) and UFRN (Federal
University of Rio Grande do Norte) for their financial support.

\section{References}

[1] Bergamaschi, F. B., and Santiago, R. H. 2014. "A Fuzzy Version of Uniformly Strongly Prime Ideals.” Presented at the 2014 IEEE Conference on Norbert Wiener in the 21st Century (21CW).

[2] Handelman, D., and Lawrence, J. 1975. "Strongly Prime Rings." Transactions of the American Mathematical Society 211: 209-23.

[3] Olson, D. 1987. "A Uniformly Strongly Prime Radical.” J. Austral. Math. Soc. (Series A) 43: 95-102.

[4] Zadeh, L. 1965. "Fuzzy Sets.” Information and Control 8: 338-53.

[5] Rosenfeld, A. 1971. "Fuzzy Groups.” Journal of Mathematical Analysis and Applications 35: 512-17.

[6] Liu, W. J. 1982. "Fuzzy Invariant Subgroups and Fuzzy Ideals.” Fuzzy Sets and Systems 8: 133-9.

[7] Jin, L. W. 1983. “Operations on Fuzzy Ideals.” Fuzzy Sets and Systems 11: 19-29.

[8] Mukherjee, T., and Sen, M. 1987. "On Fuzzy Ideals of a Ring.” Fuzzy Sets and Systems 21: 99-104.

[9] Swamy, U., and Swamy, K. 1988. "Fuzzy Prime Ideals of Rings.” Journal of Mathematical Analysis and Applications 134: 94-103.

[10] Yue, Z. 1988. "Primel-Fuzzy Ideals and Primary-Fuzzy Ideals.” Fuzzy Sets and Systems 27: 345-50.

[11] Kumar, R. 1991. "Fuzzy Nil Radicals and Fuzzy Primary Ideals.” Fuzzy Sets and Systems 43: 81-93.

[12] Kumar, R. 1991. "Fuzzy Semiprimary Ideals of Rings." Fuzzy Sets and Systems 42: 263-72.

[13] Bergamaschi, F. B., and Santiago, R. H. 2013. "Strongly Prime Fuzzy Ideals over Noncommutative Rings.” Presented at the 2013 IEEE International Conference on Fuzzy Systems (FUZZ).

[14] Navarro, G., Cortadellas, O., and Lobillo, F. 2012. “Prime Fuzzy Ideals over Noncommutative Rings.” Fuzzy Sets and Systems 199: 108-20.

[15] Lam, T. Y. 2013. A First Course in Noncommutative Rings. Springer Science \& Business Media.

[16] Parmenter, M., Passman, D., and Stewart, P. 1984. "The Strongly Prime Radical of Crossed Products.”Communications in Algebra 12: 1099-113.

[17] Malik, D. S., and Mordeson, J. N. 1991. "Fuzzy Maximal, Radical and Primary Ideals of a Ring." Information sciences 53: 237-50.

[18] McCoy, N. H. 1949. "Prime Ideal Sin General Rings." American Journal of Mathematics: 823-33. 